\title{
Cardiac cephalgia
}

\author{
Annamaria Bini · Andrea Evangelista $\cdot$ Paola Castellini · Giorgio Lambru • \\ Tullia Ferrante · Gian Camillo Manzoni · Paola Torelli
}

Received: 10 November 2008/ Accepted: 28 November 2008/Published online: 13 January 2009

(C) Springer-Verlag 2009

\begin{abstract}
The purpose of this review was to provide a critical evaluation of medical literature on so-called "cardiac cephalgia" or "cardiac cephalalgia". The 2004 International Classification of Headache Disorders codes cardiac cephalgia to 10.6 in the group of secondary headaches attributed to disorder of homoeostasis. This headache is hardly recognizable and is associated to an ischaemic cardiovascular event, of which it may be the only manifestation in $27 \%$ of cases. It usually occurs after exertion. Sometimes routine examinations, cardiac enzymes, ECG and even exercise stress test prove negative. In such cases, only a coronary angiogram can provide sufficient evidence for diagnosis. Cardiac cephalgia manifests itself without a specific pattern of clinical features: indeed, in this headache subtype there is a high variability of clinical manifestations between different patients and also within the same patient. It "mimics" sometimes a form of migraine either
\end{abstract}

A. Bini - A. Evangelista - P. Castellini - G. Lambru .

T. Ferrante · G. C. Manzoni · P. Torelli ( $\square)$

Department of Neuroscience, Headache Centre,

University of Parma, Via Gramsci 14, 43100 Parma, Italy

e-mail: paolatorelli@libero.it

A. Bini

e-mail: bini.annamaria@alice.it

A. Evangelista

e-mail: aneva78@yahoo.it

P. Castellini

e-mail: paola.castellini@libero.it

G. Lambru

e-mail: iorz@libero.it

T. Ferrante

e-mail: tullia.ferrante@live.it

G. C. Manzoni

e-mail: giancamillo.manzoni@unipr.it accompanied or not by autonomic symptoms, sometimes a form of tension-type headache; on other occasions, it exhibits characteristics that can hardly be interpreted as typical of primary headache. Pain location is highly variable. When the headache occurs as the only manifestation of an acute coronary event, the clues for suspicion are a) older age at onset, b) no past medical history of headache, c) presence of risk factors for vascular disorders and d) onset of headache under stress. Knowledge of cardiac cephalgia is scarce, due to its rare clinical occurrence and to the scant importance given to headache as a symptom concomitantly with an ischaemic cardiac event.

Keywords Cardiac cephalgia - Exertional headache . Secondary headache $\cdot$ Headache attributed to disorder of homoeostasis - Acute myocardial ischemia

\section{Introduction}

The second edition of the International Classification of Headache Disorders (ICHD-II, 2004) [1] includes forms of primary headache in which the headache symptom is the disorder, and forms of secondary headache that are attributed to underlying pathological conditions. According to this classification, to establish a diagnosis of secondary headache it is necessary to demonstrate the presence of an underlying cause or disorder, the headache must occur in close temporal relation to the demonstrated pathological condition, and it must improve or disappear within 3 months from disappearance or spontaneous remission of the causative factor.

In a study conducted on the Danish general population, investigators found that over $70 \%$ lifetime prevalence rates of secondary headaches [2]. 
While there are currently many data available about the prevalence and triggering or relieving factors of primary headaches, little is known about clinical features of secondary headaches.

In ICHD-II, cardiac cephalgia has been coded as an autonomous clinical entity to 10.6 in Group 10 ("Headache attributed to disorder of homoeostasis").

The purpose of this paper is to provide an accurate review of present-day knowledge about cardiac cephalgia.

\section{Materials and methods}

In this review of the literature, we considered only Englishlanguage articles published in scientific journals and chapters of English-language books. We searched for references by entering the key words "cardiac cephalgia" and "exertional headache" in the PubMed search tool, with no limitations to the year of publication. We first reviewed the abstracts of the publications considered for the purpose of our study in order to evaluate their relevance to the subject matter. We also reviewed the references of each publication in order to find other useful material. Overall, we included 30 cases in our review.

\section{Clinical features}

A typical clinical feature of acute coronary syndromes is oppressive pain in the chest, possibly radiating to the left arm and the neck. Headache as the only symptom of an acute cardiac event is very infrequent and is generally associated with other typical symptoms. In 1971, Sampson reported that in a group of 150 patients with angina pectoris, only $6 \%$ complained headache and this was not the only symptom that they experienced [3].

The ICHD-II diagnostic criteria for cardiac cephalgia are:

A. Headache, which may be severe, aggravated by exertion and accompanied by nausea and fulfilling criteria C-D.

B. Acute myocardial ischemia has occurred (based on the presence of ST-segment elevations or depressions and of T-wave inversions on exercise electrocardiography and the presence of elevated cardiac enzymes).

C. Headache develops concomitantly with acute myocardial ischaemia

D. Headache resolves and does not recur after effective medical therapy for myocardial ischaemia or coronary revascularization.

As it is associated to a cardiovascular event, cardiac cephalgia generally occurs after the fifth decade of life in subjects at risk for cardiovascular disease who may not have previously suffered from headache [4-11].

Unlike primary headaches that have a well-defined pain type and location, in cardiac cephalgia the clinical picture is not homogeneous.

In our review we describe the characteristics of 30 cases reported in the literature (see Table 1).

Pain is not localized in a specific area but may involve the frontal, temporal, parietal, and occipital regions. Besides, the headache may be unilateral or bilateral. Pain is almost always severe or excruciating, constrictive and oppressive or resembling migraine. In some cases there aren't accompanying symptoms, while in others pain is accompanied by autonomic phenomena such as photophobia, phonophobia, osmophobia, and nausea (30\% of cases).

Symptoms related to the underlying disorder, such as chest constriction, pain in the left arm radiating up to the mandible or epigastric pain are present in $50 \%$ of reported cases.

In $27 \%$ of the cases described in literature, cardiac cephalgia is the only manifestation of a cardiovascular ischaemic event.

The headache may start after physical exertion, even after a mild physical activity like walking. In so-called "walk headache" [4, 6, 12-14], for instance, pain appears almost instantly after starting exercise, but will disappear in a few minutes as soon as the patient stops exercising.

Cases have been reported $(33 \%)$ in which the headache appeared at rest. The frequency of this headache is highly variable; it may appear only in concomitance with the acute cardiovascular event $[10,11,15-20]$ or may be more frequent and even occur daily $[4,5,9,21]$ for periods lasting from a few weeks [5, 9, 12, 13, 22-25] to several years [6, 8, 14, 21, 26, 27].

Fifty-seven per cent of patients shows pathological alterations of the baseline ECG trace, such as ST-segment elevations or depressions and T-wave inversions [5, 8-11, 13, 15-20, 25, 27-29], as well as elevated cardiac enzymes $[5,7,10,11,16-20,26,27]$.

However, there are cases in which the ECG may look perfectly normal at rest, with pathological alterations only under stress [4, 12-14, 22-26].

This form of headache does not respond to NSAID therapy $[5,13,28]$ and triptans are contraindicated. By contrast, it responds to therapy with nitroderivatives, i.e. drugs that are used to treat cardiovascular disease.

In doubtful cases, the only test that can confirm the diagnosis of cardiac cephalgia is coronary angiography, a procedure that is capable of detecting coronary artery stenosis. Patients will then undergo an angioplasty or bypass surgery that will resolve their cardiac condition and their headache as well. Cardiac cephalgia may occur again in the event of coronary artery restenosis [7]. 


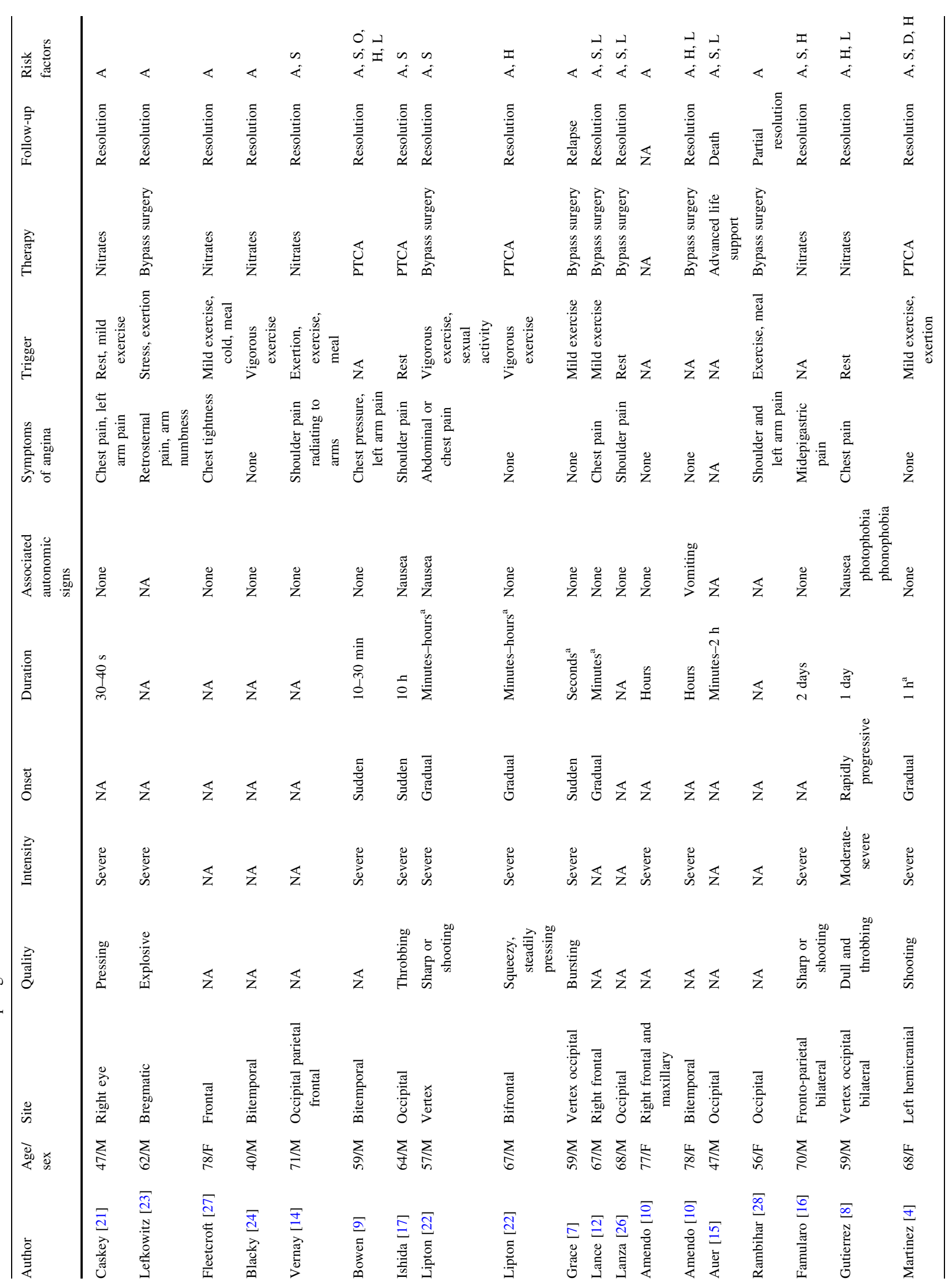




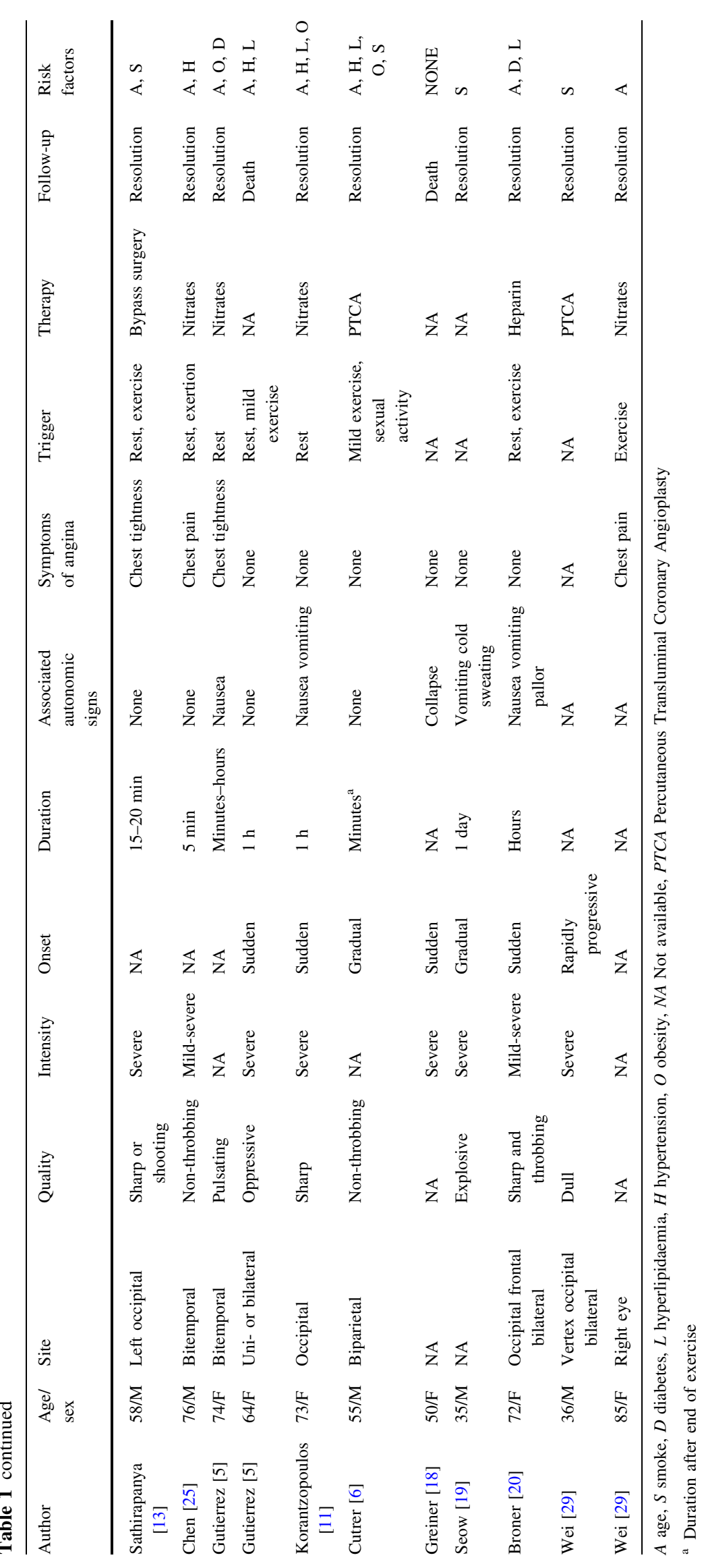




\section{Pathogenesis}

There are a variety of theories about pathogenesis of cardiac cephalgia.

The first theory is based on the fact that anginal pain is mediated by sympathetic fibres in $50-60 \%$ of cases and by vagal fibres or both in the remaining cases (10-20\% and $30-40 \%$, respectively) [30]. According to this theory, cardiac pain referred to somatic structures arises when afferent autonomic fibres (relaying visceral information from the heart through dorsal roots from C8 to T5) and somatic fibres (innervating the chest and arms) converge on sensory neurones in the spinal cord (at the posterior horns) or sympathetic trunk or at the thalamus. This convergence of autonomic sensory fibres and of trigeminal somatic fibres in the descending trigeminal nucleus is less frequent, but is nonetheless responsible for pain in the lower dental arch and in the head, and might then explain the occurrence of cardiac cephalgia. In the cases of cardiac cephalgia with pain in the occipital region, the cause could be the convergence of autonomic and somatic sensory fibres in the upper cervical spinal cord. Sympathetic and somatic sensory afferents converge into common neurones in the posterior horns of the spinal cord. Parasympathetic sensory impulses travelling trough the vagal nerve probably converge with somatic sensory impulses at the thalamus. Based on this "convergence" theory, afferent somatic and visceral fibres converge on the same neurones and therefore, when the neurones are stimulated by visceral afferents, the information for the higher centres of the central nervous system is relayed also to the corresponding somatic region $[13,16,22,30]$.

The second pathogenetic theory postulates that the sudden reduction of cardiac output associated with cardiac ischaemia increases pressure in the left ventricle and in the right atrium. The result is a reduction of venous blood flow from the brain, an elevation of intracranial pressure [22], and nociceptive distension of the intracranial structures.

The third pathogenetic theory attributes the pain of cardiac cephalgia to the release of neurochemical mediators as a result of myocardial ischaemia. These mediatorsserotonin, bradykinin, histamine, substance $\mathrm{P}$, and atrial natriuretic factor-exhibit a potent vasodilating action on the brain $[22,30,31]$.

Finally, there is a fourth pathogenetic theory assuming that cardiac cephalgia could be due to the concomitant presence of vasospasm in both coronary and cerebral vessels [32-34].

\section{Diagnosis and differential diagnosis}

Cardiac cephalgia can be suspected when a patient complains of headache in concomitance with other symptoms accompanying a cardiac ischaemic event.
In "typical" cases, to establish a diagnosis of "cardiac cephalgia" it is necessary to have a patient's past medical history demonstrating exactly the onset of headache in concomitance with acute myocardial ischaemia verified by stress tests or a radionucleotide scan. In particular, ECGs must be performed at rest and under stress in order to reveal any possible abnormalities that are typical of cardiovascular ischaemic events. An assessment of cardiac markers (CPK-MB, myoglobin, and troponin) is also useful.

Diagnosis is much more difficult when the headache occurs as the only manifestation of an acute coronary event. In such cases, the clinical clues for suspicion that may lead to an accurate diagnosis are older age at onset (which is always indicative of a secondary form), the presence of risk factors for vascular disorders, and onset of headache under stress.

Therefore, it is important to differentiate between cardiac cephalgia as the only manifestation of an acute coronary syndrome and an attack of migraine without aura. Migraine occurs between the second and the third decade of life is more frequent in women, and often develops around the time of the first menstrual cycle. It is often preceded by warning symptoms, such as mood change, shivering, yawning, and hunger. The headache phase is represented by a progressively worsening headache, which usually appears when the patient awakes in the morning and can be triggered by hormonal factors, weather changes, travel, or stressful events. Pain intensity increases progressively for a few hours until it peaks and remains stable throughout the attack.

Both migraine and cardiac cephalgia may occur as a severe headache accompanied by autonomic phenomena, most notably nausea, and can both be worsened by exercise or may develop at rest. It is extremely important to differentiate this condition from other forms, especially migraine without aura. In fact, vasoconstricting agents (such as triptans or ergot derivatives) are indicated for treatment of migraine but should be avoided in patients with cardiac ischaemia because they would worsen their clinical condition.

Triptans are selective agonists of the 5-HT1B-1D receptors localized in meningeal arteries, perivascular trigeminal nerve terminals and the central nervous system. They carry out their anti-migraine action by constricting extracerebral intracranial arteries and by inhibiting peripheral neuronal activity and neurotransmission by second-order neurones of the trigeminal-cervical complex $[35,36]$. Although these drugs exhibit their vascular effect mainly at the cranial level, a minimal vasoconstricting effect was also observed in the coronary artery district. For that reason, they are not indicated in patients with cardiovascular disease [37, 38]. 
Other differential diagnoses with cardiac cephalgia, when there are no accompanying symptoms typical of myocardial ischaemia, are primary and secondary forms of exertional headache, thunderclap headache, orgasmic headache, and cough headache. A common feature of this group of headaches is the sudden onset of pain. Primary, or benign, cough headache is precipitated by coughing, is bilateral, and usually resolves in $5 \mathrm{~min}$. Primary, or benign, exertional headache may be precipitated by any form of exercise, is usually bilateral and pulsating, and lasts from a few minutes to $24 \mathrm{~h}$. The orgasmic subtype of primary, or benign, headache associated with sexual activity is characterized by sudden severe pain occurring at orgasm. Apart from an accurate evaluation of the patient's past medical history, it is always necessary to perform a brain MRI to exclude any underlying pathological conditions.

Another differential diagnosis should be with headache triggered by angina treatment such as nitroglycerine, which may precipitate a headache or a migraine attack with its vasodilating action.

Nitric oxide (NO) donor-induced headache is a form of secondary headache that appears following administration of nitroderivatives. Both headache and non-headache patients may develop pain soon after receiving medication (within $10 \mathrm{~min}$ ). This headache is bilateral, throbbing and exacerbated by physical activity, and it disappears after about $1 \mathrm{~h}$ from medication intake. Late NO donor-induced headache occurs more frequently in headache patients. It appears with the same features as primary headache and disappears about $72 \mathrm{~h}$ after medication intake [1]. In this case, for differential diagnosis between cardiac cephalgia and NO donor-induced headache, it is important to determine the temporal relation between the administration of nitroderivatives and the appearance of cranial pain in patients with a verified or suspected diagnosis of acute ischaemic coronary disease.

Failure to recognize and correctly diagnose cardiac cephalgia may have severe consequences.

In cases of de novo headache in patients over 50 years of age at risk for cardiovascular disease, some authors believe that it is necessary to perform an ECG and an assessment of cardiac markers [15, 16].

\section{Conclusion}

Headache is a non-specific symptom of various conditions and is rarely considered in medical textbooks as an accompanying symptom of acute coronary syndromes.

In all likelihood, then, cardiac cephalgia is an underestimated symptom in emergency departments but also in neurology or internal medicine departments, where physicians probably have little knowledge of this subtle form of headache.

Conflict of interest None.

\section{References}

1. Headache Classification Subcommittee of the International Headache Society (2004) The international classification of headache disorders: 2nd edn. Cephalalgia 24(suppl 1):1-160

2. Rasmussen BK, Olesen J (1992) Symptomatic and non symptomatic headache in a general population. Neurology 42:12251231

3. Sampson JM (1971) Pathophysiology and differential diagnosis of cardiac pain. Prog Cardiovascular Dis 13:507-531

4. Martínez HR, Rangel-Guerra RA, Cantú-Martínez L, GarzaGómez J, González HC (2002) Cardiac headache: hemicranial cephalalgia as the sole manifestation of coronary ischemia. Headache 42:1029-1032

5. Gutiérrez Morlote J, Fernández García JM, Timiraos Fernández JJ, Llano Cardenal M, Rodríguez E, Pascual Gómez J (2005) Cardiac cephalgia: an under diagnosed condition? Rev Esp Cardiol 58:1476-1478

6. Cutrer FM, Huerter K (2006) Exertional headache and coronary ischemia despite normal electrocardiographic stress testing. Headache 46:165-178

7. Grace A, Horgan J, Breathnach K, Staunton H (1997) Anginal headache and its basis. Cephalalgia 17:195-196

8. Gutiérrez-Morlote J, Pascual J (2002) Cardiac cephalgia is not necessarily an exertional headache: case report. Cephalalgia 22:765-766

9. Bowen J, Oppenheimer G (1993) Headache as a presentation of angina: reproduction of symptoms during angioplasty. Headache 33:238-239

10. Amendo MT, Brown BA, Kossow LB, Weinberg RB (2001) Headache as the sole presentation of acute myocardial infarction in two elderly patients. Am J Geriatr Cardiol 10:100-101

11. Korantzopoulos P, Karanikis P, Pappa E, Dimitroula V, Kountouris E, Siogas K (2005) Acute non-St-elevation myocardial infarction presented as occipital headache with impaired level of consciousness. Angiology 56:627-630

12. Lance JW, Lambros J (1998) Unilateral exertional headache as a symptom of cardiac ischemia. Headache 38:315-316

13. Sathirapanya P (2004) Anginal cephalgia: a serious form of exertional headache. Cephalalgia 24:231-234

14. Vernay D, Deffond D, Fraysse P, Dordain G (1989) Walking headache: an unusual manifestation of ischemic heart disease. Headache 29:350-351

15. Auer J, Berent R, Lassnig E, Eber B (2001) Headache as a manifestation of fatal myocardial infarction. Neurol Sci 22:395-397

16. Famularo G, Polchi S, Tarroni P (2002) Headache as a presenting symptom of acute myocardial infarction. Headache 42:1025-1028

17. Ishida A, Sunagawa O, Touma T, Shinzato Y, Kawazoe N, Jukiyama K (1996) Headache as a manifestation of myocardial infarction. Jpn Heart J 37:261-263

18. Greiner F, Rothrock J (2006) Thunderclap headache, cardiopulmonary arrest, and myocardial infarction. Headache 46(3):512

19. Seow VK, Chong CF, Wang TL, Ong JR (2007) Severe explosive headache: a sole presentation of acute myocardial infarction in a young man. Am J Emerg Med 25:250-251

20. Broner S, Lay C, Newman L, Swerdlow M (2007) Thunderclap headache as the presenting symptom of myocardial infarction. Headache 47:724-733 
21. Caskey WH, Spierings ELH (1978) Headache and heartache. Headache 18(5):240-243

22. Lipton RB, Lowenkopf T, Bajwa ZH, Leckie RS, Ribeiro S, Newman LC, Greenberg MA (1997) Cardiac cephalgia: a treatable form of exertional headache. Neurology 49:813-816

23. Lefkowitz D, Biller J (1982) Bregmatic headache as a manifestation of myocardial ischemia. Arch Neurol 39:130

24. Blacky RA, Rittelmayer JT, Wallace MR (1987) Headache angina. Am J Cardiol 60:730

25. Chen SP, Fuh JL, Yu WC, Wang SJ (2004) Cardiac cephalalgia: case report and review of the literature with new ICHD-II criteria revisited. Eur Neurol 51:221-226

26. Lanza GA, Sciahbasi A, Sestito A, Maseri A (2000) Angina pectoris: a headache. Lancet 356:998

27. Fleetcroft R, Maddocks JL (1985) Headache due to ischaemic heart disease. J R Soc Med 78:676

28. Rambihar VS (2001) Headache angina. Lancet $357: 72$

29. Wei JH, Wang HF (2008) Cardiac cephalalgia: case reports and review. Cephalalgia 28:892-896

30. Meller ST, Gebhart GF (1992) A critical review of the afferent pathways and the potential chemical mediators involved in cardiac pain. Neuroscience 48:501-524
31. Petersen JA, Nielsen FE (2002) Headache: a rare manifestation of angina pectoris. Ugeskr Laeger 164(19):2515-2516

32. Ramadan NM (1996) Headache caused by raised intracranial pressure and intracranial Hypotension. Curr Opin Neurol 9:214-218

33. McCrory P (1997) Recognizing exercise-related headache. Physician Sports Med 25:33-43

34. Silbert PL, Hankey GJ, Prentice DA (1989) Angiographically demonstrated arterial spasm in a case of benignal sexual headache end benign exertional headache. Aust N Z J Med 19:466-468

35. Goadsby PJ (2000) The pharmacology of headache. Prog Neurobiol 62:509-525

36. Goadsby PJ, Lipton RB, Ferrari MD (2002) Migraine-current understanding and treatment. N Engl J Med 346:257-270

37. Nilsson T, Longmore J, Shaw D, Pantev E, Bard JA, Branchek T, Edvinsson L (1999) Characterisation of 5-HT receptors in human coronary arteries by molecular and pharmacological techniques. Eur J Pharmacol 372:49-56

38. Newman CM, Starkey I, Buller N, Seabra-Gomes R, Kirby S, Hettiarachchi J, Cumberland D, Hillis WS (2005) Effects of sumatriptan and eletriptan on diseased epicardial coronary arteries. Eur J Clin Pharmacol 61:733-742 